Review

\title{
Multiscale modeling in food engineering
}

\section{A R T I C L E I N F O}

\section{Article history:}

Received 29 March 2012

Received in revised form 13 August 2012

Accepted 18 August 2012

Available online $\mathrm{xxxx}$

\section{Keywords:}

Multiscale

Modelling

Transport phenomena

Microstructure

Tomography

Lattice Boltzmann

\begin{abstract}
A B S T R A C T
Since many years food engineers have attempted to describe physical phenomena such as heat and mass transfer that occur in food during unit operations by means of mathematical models. Foods are hierarchically structured and have features that extend from the molecular scale to the food plant scale. In order to reduce computational complexity, food features at the fine scale are usually not modeled explicitly but incorporated through averaging procedures into models that operate at the coarse scale. As a consequence, detailed insight into the processes at the microscale is lost, and the coarse scale model parameters are apparent rather than physical parameters. As it is impractical to measure these parameters for the large number of foods that exist, the use of advanced mathematical models in the food industry is still limited. A new modeling paradigm - multiscale modeling - has appeared that may alleviate these problems. Multiscale models are essentially a hierarchy of sub-models which describe the material behavior at different spatial scales in such a way that the sub-models are interconnected. In this article we will introduce the underlying physical and computational concepts. We will give an overview of applications of multiscale modeling in food engineering, and discuss future prospects.
\end{abstract}

(c) 2012 Published by Elsevier Ltd.

\section{Contents}

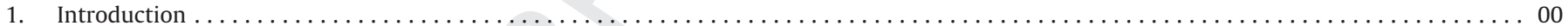

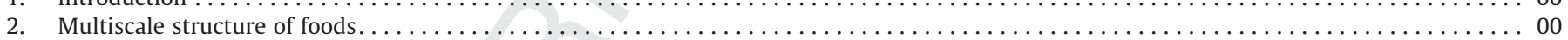

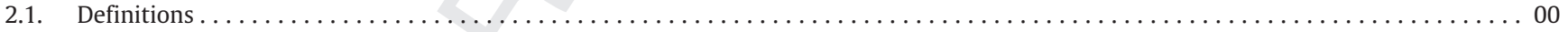

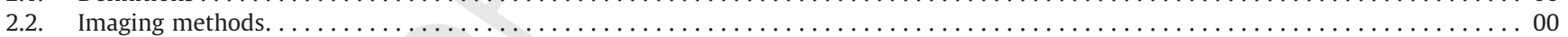

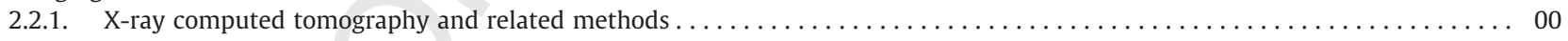

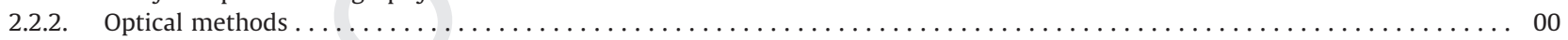

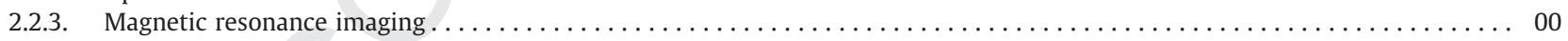

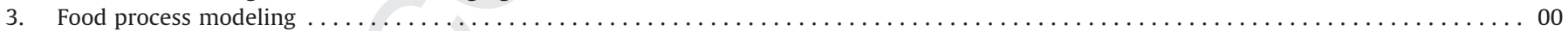

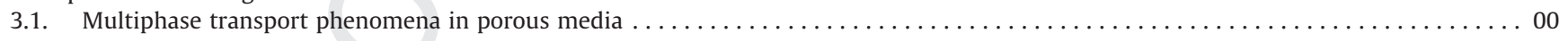

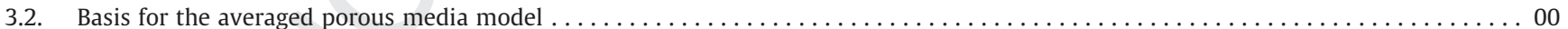

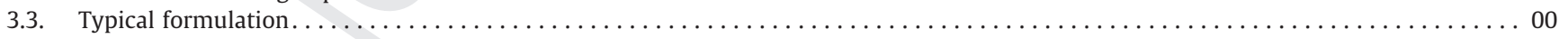

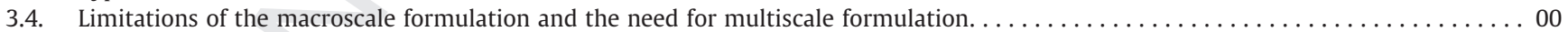

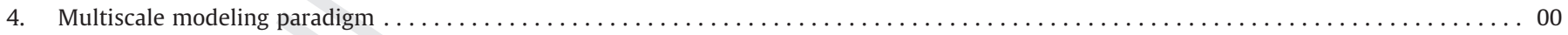

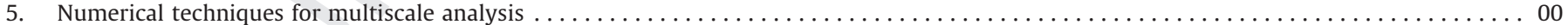

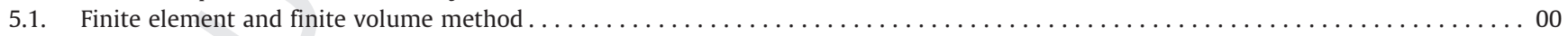

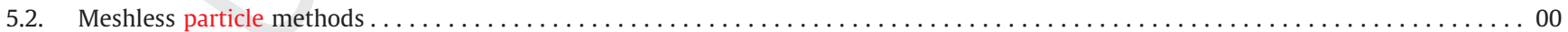

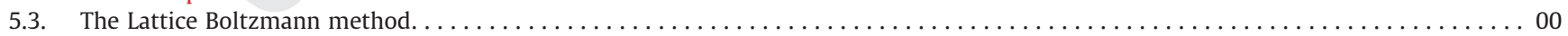

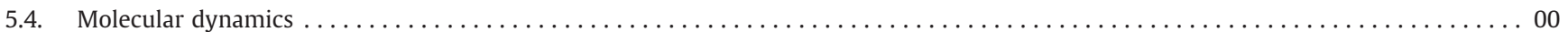

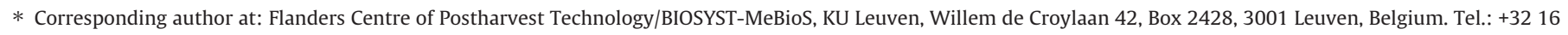
322375; fax: +32 16322955 .

E-mail address: bart.nicolai@biw.kuleuven.be (B.M. Nicolaï).

0260-8774/\$ - see front matter @ 2012 Published by Elsevier Ltd. http://dx.doi.org/10.1016/j.jfoodeng.2012.08.019 


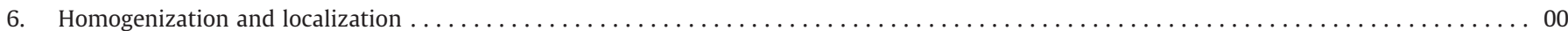

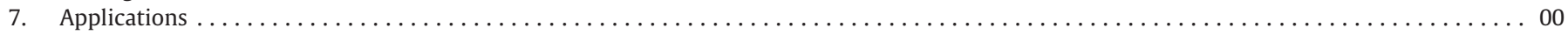

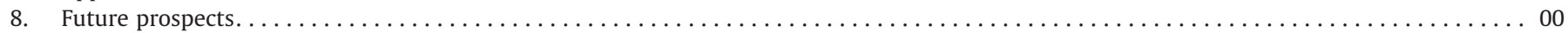

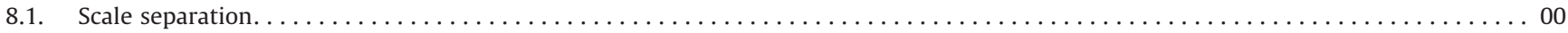

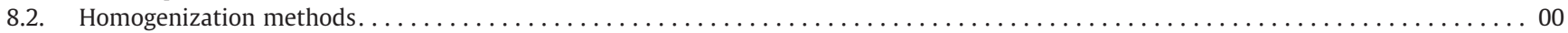

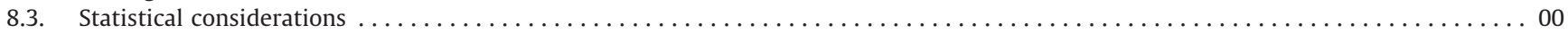

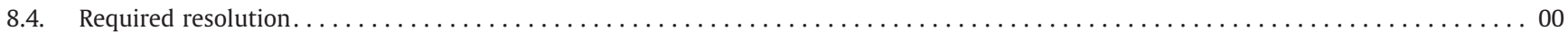

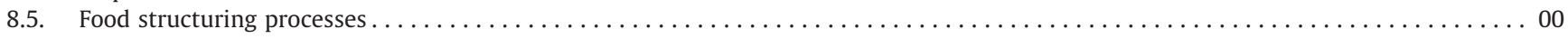

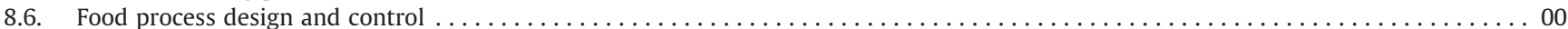

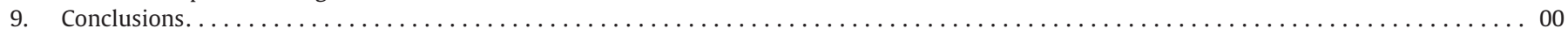

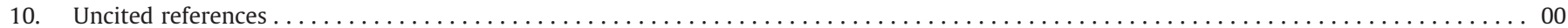

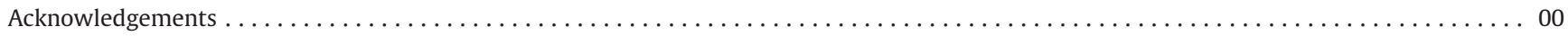

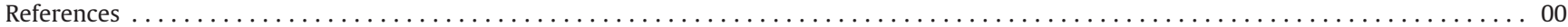

\section{Introduction}

Since the early work of Ball (1923) to model heat transfer during sterilization, food engineers have attempted to develop mathematical models of food processes, either for improving their understanding of the physical phenomena that occur during food processing, or for designing new or optimizing existing food processes (Datta, 2008; Perrot et al., 2011; Sablani et al., 2007). Depending on the complexity, different modeling approaches are used that can range from being completely observation-based to completely physics-based: simple relationships between variables such as sweetness as perceived by a human expert and the sugar content of the food are typically described using polynomial models; variables that vary as a function of time, such as the inactivation of micro-organisms during pasteurization, are modeled using ordinary differential equations; and variables that depend on both time and space, such as the temperature and moisture field inside a potato chip during frying are described by means of partial differential equations of mathematical physics (for a more extensive review of these and other modeling concepts, see Datta, 2008; Perrot et al., 2011; Sablani et al., 2007). The latter are difficult to solve: except for trivial geometries and boundary conditions usually no closed form analytical solution is known, and numerical techniques are required to compute an approximate solution of the governing equations. Finite element and finite volume methods are amongst the most popular numerical methods for solving partial differential equations, and several computer codes are commercially available for solving problems such as conduction and convective heat transfer, (visco)elastic deformation, fluid flow and moisture diffusion (e.g., ANSYS (www.ansys.com), Comsol Multiphysics (www.comsol.com), Abaqus (www.simulia.com)). All commercial codes have preprocessing facilities that allow defining complicated geometries, and most of them can be adapted to the needs of the process engineer through user routines. As often physical processes are inherently coupled, e.g., heat and mass transfer, hygro- or thermoelastic deformation, many of these codes also provide so-called multiphysics capabilities.

A mathematical model is only complete when the boundary conditions are specified and the material properties are known. Boundary conditions are either imposed or are design variables to be optimized; material properties need to be known in advance. As engineers in other disciplines often work with a limited number of materials, commercial codes typically include libraries of material properties that are sufficient for many engineering applications. However, this is not the case for food engineering: not only is the number of different foods vast, recipes vary and new foods are created every day. While engineering properties have been measured carefully for a variety of common foods (see, e.g., Rao et al., 2005; Sahin and Sumnu, 2006), for the majority of foods this is not the case. Many food engineers have, therefore, attempted to predict proper- ties based on chemical composition and microstructure. Especially the latter typically has a large effect on the physical behavior of the food. The many correlations that express the thermal conductivity as a function of the food composition and microstructure are a good example (Becker and Fricke, 1999; Fikiin and Fikiin, 1999; van der Sman, 2008b). The correlations often rely on assumptions that are non-trivial. For example, the direction of heat flow compared to the microstructural organization of the food (parallel, perpendicular, or a mixture of both) has a large effect on the estimation of the thermal conductivity; while for some products such as meat this is often obvious, for other products this is far less clear. Other authors have used averaging procedures: they first derived governing equations that took into account often simplified microstructural features, and then averaged them spatially to obtain equations that contained effective or apparent material properties that embodied microstructural features (e.g., Datta, 2007a,b; Ho et al., 2008; Whitaker, 1977). The process design is then entirely based on the latter equations without further reference to the microstructure. Another approach is to solve the governing model at the resolution of the underlying microstructure. However, in order to predict variables at the food process scale this would require computer resources that are far beyond the current capabilities. Also, materials are hierarchically structured: beyond the microscale there are probably further relevant layers of complexity with an ever increasing resolution, making the problem even more difficult to solve.

A new modeling paradigm, called multiscale modeling, has emerged in other branches of science and engineering to cope with this. Multiscale models are basically a hierarchy of sub-models which describe the material behavior at different spatial scales in such a way that the sub-models are interconnected. The advantage is that they predict macroscale behavior that is consistent with the underlying structure of matter at different scales while not requiring excessive computer resources. Also, while incorporating smaller scales into the model, less assumptions are required for the material properties, which tend towards physical constants that are well known, or constitutive equations at the expense of increasing the geometrical complexity. Finally, the effect of macroscale behavior on microscale phenomena can be evaluated as well.

In this article we will discuss the potential of multiscale modeling in food process engineering. The focus will be on multiscale behavior in the spatial domain rather than in the time domain, although both are coupled: events at very small scales (e.g., molecular collisions) typically occur in very short time intervals, whereas time constants for macroscopic events at the process scale (e.g., heat transfer in a can) are much larger. Multiscale phenomena in the time domain are usually dealt with by uncoupling equations based on time constant considerations, adaptive time stepping schemes or stiff systems solvers.

The article is organized as follows. We will first discuss some experimental techniques that can be used to obtain geometrical 


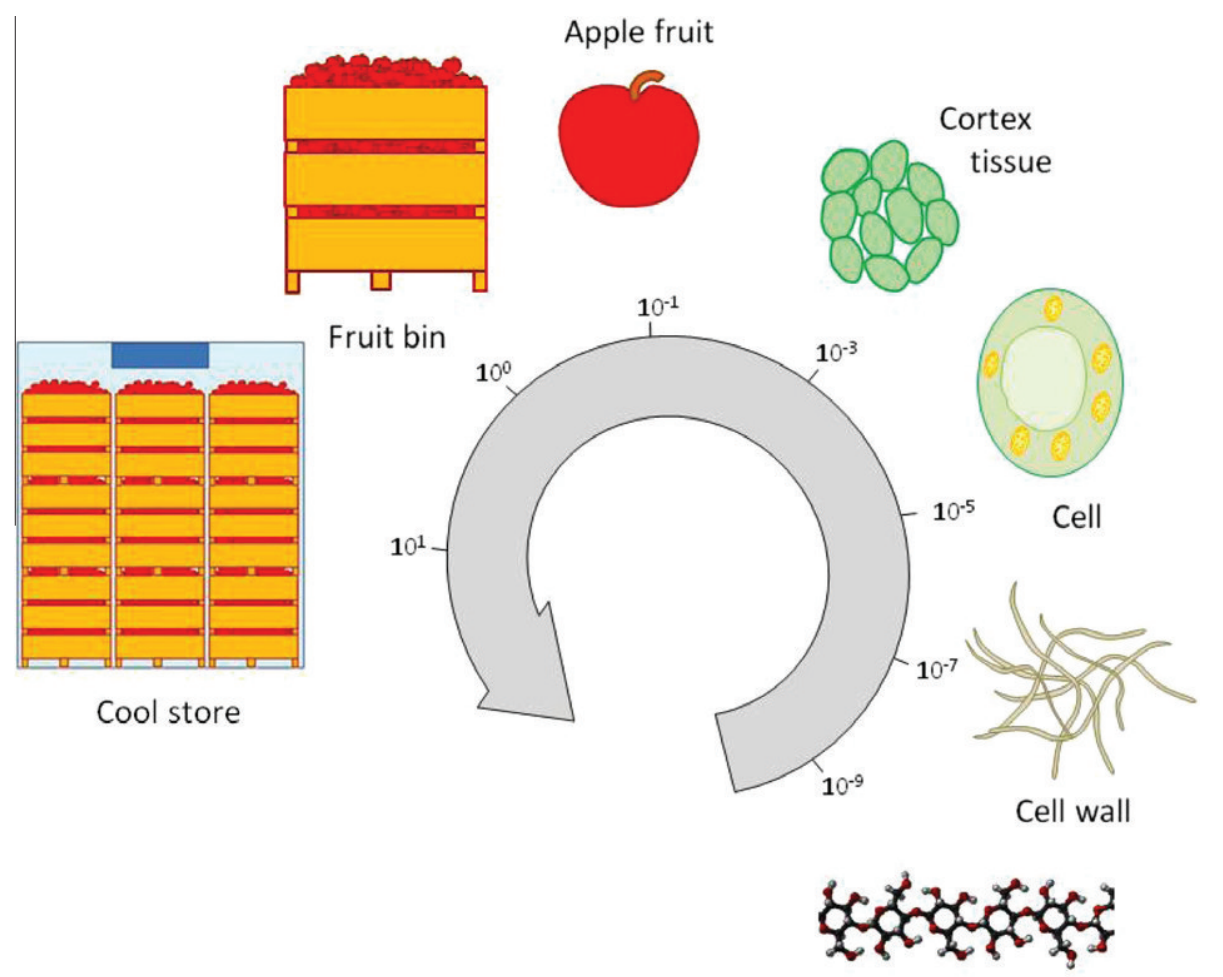

Cellulose molecule

Fig. 1. Multiscale aspects of moisture loss during apple storage.

models of the food at different spatial scales, with an emphasis on $\mathrm{X}$-ray computed tomography at different resolutions. We will then shortly discuss some physical processes in food engineering that are well suited for multiscale modeling. We will show that multiscale problems may include different physics: at very small scales the continuum hypothesis breaks down and discrete simulation methods are required. We will pay particular attention to connecting the different scales, especially when different types of physics are involved. Finally we will discuss some examples of multiscale modeling in food process engineering and give some guidelines for future research.

\section{Multiscale structure of foods}

\subsection{Definitions}

According to the Merriam-Webster online dictionary (Anonymous, 2012), structure is 'something arranged in a definite pattern of organization', or 'the arrangement of particles or parts in a substance or body'. In most materials including foods, structure spans many scales. For example, an apple consists of different tissues (epidermis, inner and outer cortex, vascular tissue) that are the constituent elements of its structure (Fig. 1). If we observe a tissue with a light microscope, its cellular nature reveals itself. Further, cells have features such as cell walls, plastids that are at least an order of magnitude smaller. These features can further be decomposed into their constituent biopolymers at dimensions of the order of $1 \mathrm{~nm}$. At the other side of the scale, apples can be put in boxes, and boxes in cool stores with a typical characteristic length of $10 \mathrm{~m}$. Physical phenomena such as moisture loss - an important variable of concern in the design of cool stores - occur at all scales mentioned, thereby spanning 10 orders of magnitude. Foods are thus truly multiscale materials.

Changes in the structure of the food at the microscale or beyond during storage and processing can be significant and affect the macroscopic appearance, quality and perception of food (Aguilera,

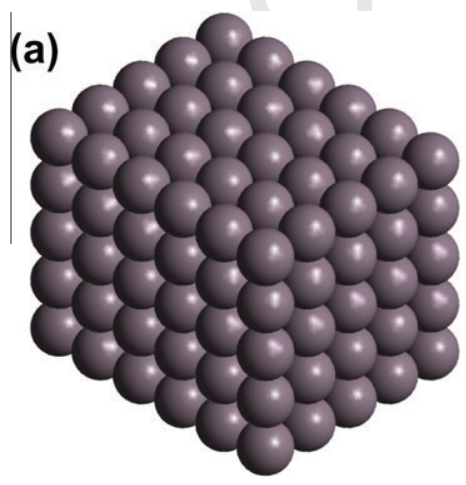

(b)

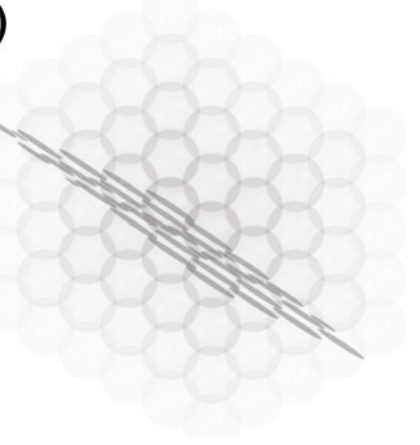

(c)

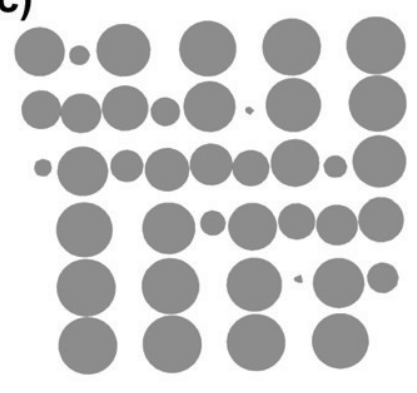

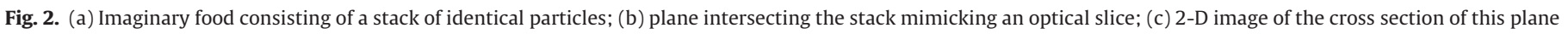
with the stack. Although the diameter of all particles is equal, that of the circles obtained where the plane intersects the spheres is not. 
2005). Due to the complexity of this multiscale structure of foods, straightforward methodologies that link its macroscale properties to changes of the microscale features do not exist today, as opposed to many engineering materials with a well-ordered microstructure, for which the relationship with macroscopic properties can be easily understood based on fundamental physics. Multiscale models can serve this purpose.

For further use in this article we will now define the following (to some extent arbitrary) scales:

- Food plant scale $\left(1-10^{3} \mathrm{~m}\right)$ : the scale of food plant equipment, including retorts, cool stores, extruders, UHT units etc.

- Macroscale $\left(10^{-3}-10^{0} \mathrm{~m}\right)$ : discrete foods or food ingredients that can be observed and measured by the naked eye, from a single wheat grain to a baguette

- Microscale $\left(10^{-6}-10^{-3} \mathrm{~m}\right)$ : food features such as air pores, micro capillaries, cells, fibers that need light microscopy to be visualized

- Mesoscale $\left(10^{-7}-10^{-6} \mathrm{~m}\right)$ : food structures such as cell walls and emulsions

- Nanoscale $\left(10^{-9}-10^{-7} \mathrm{~m}\right)$ : food biopolymers

Obviously this terminology is somewhat arbitrary and scales may overlap in practice. Some authors use the term microscale for everything that is smaller than the macroscale. In this article, we will also use the terms coarse and fine scale when only relative dimensions are important.

\subsection{Imaging methods}

A first step in multiscale modeling is often to visualize the structure of foods at multiple scales and to construct a geometric model that can be used for further analyses. Several techniques are available, including CCD cameras, optical microscopy in the visual and (near)-infrared wavelength range of the electromagnetic spectrum, transmission and scanning electron microscopy, atomic force microscopy. These techniques are well known and the reader is referred to the literature for more details (Aguilera, 2005; Russ, 2004). However, the majority of these techniques produce geometrical information that is essentially 2-D. In many cases this is not sufficient. Consider, for example, an imaginary food consisting of a stack of identical particles (Fig. 2a). If we take a cross section with random orientation through the stack simulating what we would do in preparing a slice for light microscopy (Fig. 2b), we obtain a collection of circles with various unequal radii (Fig. 2c). This would, wrongly, suggest that the food is composed of differently sized particles. Further, the porosity would also depend on the orientation of the cross section. The most important artifact, however, would be that there are 2-D cross sections in which all pores are unconnected, while in 3-D there is a full connectivity. This would have, for example, major consequences on our understanding of mass transport phenomena through the pore space. We will, therefore, discuss only methods that provide 3-D images of foods that can be converted to solid models appropriate for numerical discretization of multiphysics models. More specifically, we will focus on X-ray computed tomography, optical methods and magnetic resonance imaging.

\subsubsection{X-ray computed tomography and related methods}

X-ray computed tomography (CT) was developed in the late 1970s to visualize the internal structure of objects non-destructively. These first, mainly medical, CT scanners had a pixel resolution in the order of $1 \mathrm{~mm}$. In the 1980s, after some technological advances towards micro-focus X-ray sources and high-tech detection systems, it was possible to develop a micro-CT (or $\mu \mathrm{CT}$ ) system with nowadays a pixel resolution 1000 times better than the medical CT scanners. The technique of X-ray (micro)-CT is based

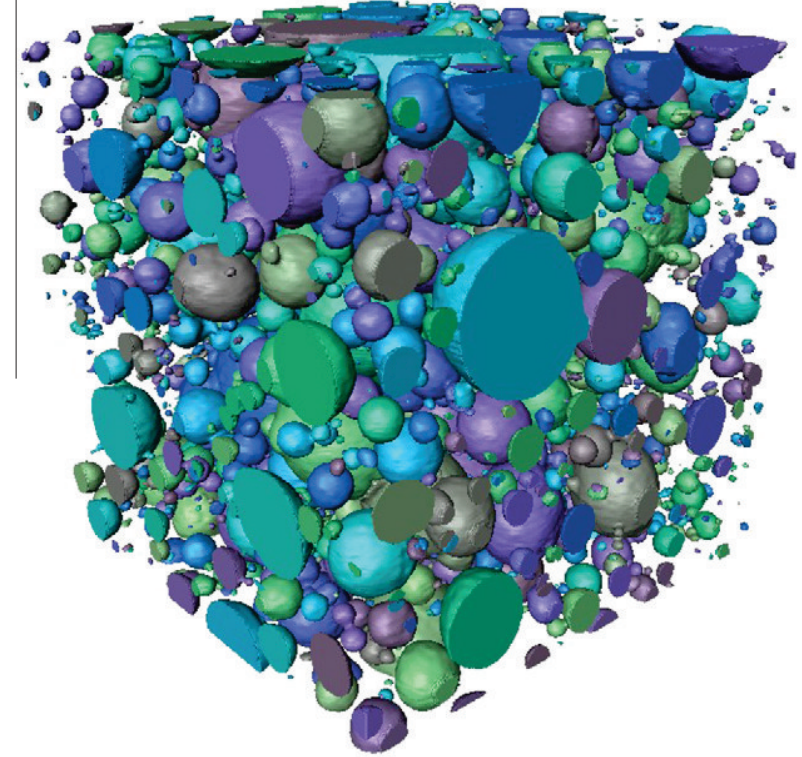

Fig. 3. 3-D micro CT image of a sugar foams consisting of sugar, agar and water obtained on a SkyScan 2011 benchtop X-ray nano CT with a pixel resolution of $500 \mathrm{~nm}$ (E. Herremans, KU Leuven, unpublished).

on the interaction of X-rays with matter. When X-rays pass through an object they will be attenuated in a way depending on the density and atomic number of the object under investigation and of the used X-ray energies. By using projection images obtained from different angles a reconstruction can be made of a virtual slice through the object. When different consecutive slices are reconstructed, a 3-D virtual representation of the object can be obtained, which provides qualitative and quantitative information about its internal structure. Such information is useful for numerical analysis of these porous structures: it can be used to generate geometric CAD models for numerical analysis based on a parametric description of the geometry of the material (e.g., porosity, pore distribution), or by directly using the 3-D images for generation of such models (Mebatsion et al., 2008; Moreno-Atanasio et al., 2010). The reconstructed 3-D volume is typically a data stack of 2-D images with sizes up to several Gigabytes for one CT scan. X-ray $\mathrm{CT}$ is the only technology to date that covers a large range of scales - currently from about $200 \mathrm{~nm}$ up to $20 \mathrm{~cm}$ and more.

Several examples of X-ray CT for food are discussed by Falcone et al. (2006). X-ray micro-CT has been successfully used to visualize, amongst others, foams (Lim and Barigou, 2004), bread (Falcone et al., 2004), apple (Mendoza et al., 2007), processed meat (Frisullo et al., 2009), chicken nuggets (Adedeji and Ngadi, 2011), biscuits (Frisullo et al., 2010) and coffee (Frisullo et al., 2012).

Rather recently, lab-based nano CT systems have been introduced opening up a new era in X-ray imaging with a spatial resolution below 1 micrometer (Hirakimoto, 2001), even down to some hundreds of nanometers. Realizing submicron pixel sizes requires increased performance of the X-ray source, rotation stage and Xray detector. Before, submicron resolutions could only be obtained at synchrotron X-ray facilities, which are not that readily accessible for researchers. Synchrotron radiation micro-CT with submicron resolution has been applied successfully to foods such as apple and pear (Verboven et al., 2008). In Fig. 3 an image of a foam obtained with a bench top nano CT machine is shown at $500 \mathrm{~nm}$ resolution.

Even higher resolutions of up to $15 \mathrm{~nm}$ are possible with soft $X$-ray tomography. Soft X-rays are typically produced by synchrotrons or laser-produced plasma's. Soft X-ray tomography has been used for visualizing cellular architecture (Larabell and Nugent, 
2010) but has limited penetration depth (typically $<10 \mu \mathrm{m}$ ). Similar to X-ray tomography and microscopy, electron tomography uses a tilted stage in combination with a transmission electron microscope to acquire transmission images at various angles that are then reconstructed to a 3-D model with a resolution down to 5 $20 \mathrm{~nm}$. As far as the authors are aware of there are no applications in food science yet.

\subsubsection{Optical methods}

In confocal laser scanning microscopy, points are illuminated one by one by a laser, and the fluorescence is measured through a pinhole to eliminate out of focus light. The object is scanned point by point, and 3-D images may be constructed by moving the focal plane inside the object. However, the penetration depth is limited to a few hundred micrometers or less, depending on the optical properties of the specimen and the actual optical setup (Centonze and Pawley, 2006). Optical Coherence Tomography (OCT) is a relatively recent contactless high-resolution imaging technique, which has been introduced for biomedical diagnostics applications such as the detection of retinal diseases. In OCT, the sample is typically illuminated with light in the near infrared. The backscattered and - reflected photons from the sample are collected and brought to interfere with a reference beam. From the interference pattern the location of the scattering sites within the sample can be determined. The penetration depth is several times higher than that obtained with, e.g., confocal microscopy. Since OCT detects inhomogeneities in the refractive index of materials, the images it produces are complementary to those obtained with, e.g., X-ray CT where the contrast is related to the density distribution. Meglinski et al. (2010) used OCT to monitor defects and rots in onion.

\subsubsection{Magnetic resonance imaging}

In magnetic resonance imaging (MRI), magnetic nuclei such as protons are aligned with an externally applied magnetic field. This alignment is subsequently perturbed using an alternating magnetic field and this causes the nuclei to produce a rotating magnetic field detectable by the scanner. The signal is spatially encoded using magnetic field gradients and is afterwards reconstructed into a 3-D image (Hills, 1995). MRI is particularly suitable for high water content foods. Typical spatial resolutions are 10$50 \mu \mathrm{m}$ (slice thickness $100-1000 \mathrm{~mm}$ ) and thus considerably less than X-ray micro and nano CT, but the contrast is usually much better in biological tissues and different substances (water, oil, sugar) can be distinguished (Clark et al., 1997). MRI has been used to visualize internal quality defects of fruit such as voids, worm damage or bruising and their variation over time (Chen et al., 1989; McCarthy et al., 1995; Lammertyn et al., 2003), meat structure (Collewet et al., 2005), bread microstructure (Ishida et al., 2001) and a plethora of other applications, but its main power is in 3-D mapping of transport of heat and mass in foods (e.g., Verstreken et al., 1998; Rakesh et al., 2010).

\section{Food process modeling}

Food process modeling is an essential tool to understand, design and control food processes (Datta, 2008; Perrot et al., 2011; Sablani et al., 2007). We will focus here on transport phenomena as they are arguably the most important processes in food unit operations. We will show how difficulties with modeling these phenomena lead to the need for a multiscale approach.

\subsection{Multiphase transport phenomena in porous media}

Modeling of transport phenomena applied to food processes at the macroscale can be broadly divided into those for single phase and those for multiphase. Since multiphase models, particularly when the solid phase is included, can cover the vast number of food processes, discussion in this section will be restricted to multiphase porous media-based transport models. The multiphase porous media-based approach at the macroscale incorporating averaged material properties appears to be the most popular among the detailed mechanistic approaches to model food processes. It has been used to model a number of food processes, including drying (Lamnatou et al., 2010), rehydration (Weerts et al., 2003), baking (Ni and Datta, 1999; Zhang et al., 2005), frying (Halder et al., 2007; Yamsaengsung and Moreira, 2002), meat cooking (Dhall and Datta, 2011), microwave heating (Ni et al., 1999), gas transport (Ho et al., 2008) and microwave puffing (Rakesh and Datta, accepted for publication). While these examples use distributed evaporation, evaporation at a sharp front combined with the same macroscale formulation has also been applied to a number of food processes (Farid, 2002).

The multiphase models of food processes, however, cover a wide range as to how mechanistic the approaches are. For example, frying has been modeled as completely empirical (lumped parameter) all the way to multiphase, multicomponent and multimode transport in the porous media model (the topic of this section). Such detailed models, although around for some years in food (e.g., Ni et al., 1999), have not become commonplace primarily due to the complexity of the computations and the unavailability of detailed transport properties for food materials that are needed for such models.

\subsection{Basis for the averaged porous media model}

Description of fluid flow and transport in a porous medium by considering it in an exact manner (i.e., solving Navier-Stokes equations for fluids in the real pore structure) is generally intractable at least at the macroscale (Bear, 1972) due to the geometry of the intricate internal solid surfaces that bound the flow domain, although this is precisely what is pursued for small dimensions at the microscale (Keehm et al., 2004), as described later. For porous mediabased modeling of food processing problems, most of the studies have been at the macroscale. A macroscale continuum-based porous media transport model (as described in the following section) consists of transport equations with the variables and parameters averaged over a representative elementary volume (REV). The size of this $\mathrm{REV}$ is large compared to the dimension of the pores or solid particle structure but small compared to the dimensions of the physical domain of interest (e.g., an apple fruit). The size of the REV can vary spatially and depends on the quantity of interest (i.e., permeability). Using Lattice-Boltzmann simulation, Zhang et al. (2000) showed that the quantity of interest fluctuates rapidly as the scale gets smaller but approaches a constant value with increasing scale. Thus, they defined a statistical REV as the volume beyond which the parameter of interest becomes approximately constant and the coefficient of variation (standard deviation divided by the mean) is below a certain desired value. Through such averaging, the actual multiphase porous medium is replaced by a fictitious continuum; a structureless substance (Bear, 1972), also called a smeared model or a homogeneous mixture model, where neither the geometric representation of the pore structure nor the exact locations of the phases are available. Details of porous media models can be found in several textbooks (e.g., Bear, 1972; Schrefler, 2004; Vafai, 2000).

\subsection{Typical formulation}

Food process models that are based on multiphase transport in a porous medium have typically used the common volume averaged equations (Whitaker, 1977), although the linkage to the averaging process may not always be made explicit. The food ma- 
trix is mostly considered rigid although deformable porous media have been considered - the relevant equations are provided in detail in Datta (2007a,b) and Dhall and Datta (2011). The phases considered for a solid food are the solid, liquid (e.g., water, oil), and gas (e.g., water vapor, carbon dioxide, nitrogen, ethylene). Evaporation is considered either distributed throughout the domain or at an evaporating interface and is dictated by the local equilibrium between the liquid and vapor phase. Transport mechanisms considered are capillarity and gas pressure (due to evaporation) for liquid transport, and molecular diffusion and gas pressure for vapor and air transport. Pressure driven flow is modeled using Darcy's law when the permeability is small (pores are small, including possible Knudsen effects; Tanikawa and Shimamoto, 2009) or its more general Navier-Stokes analog when the matrix is very permeable (Hoang et al., 2003; Nahor et al., 2005). Local thermal equilibrium, where all phases share the same temperature at a location, is often assumed, leading to one energy equation. The final governing equations for a rigid matrix consist of one energy equation, one mass balance equation and either the Darcy's law or the Navier-Stokes for the momentum equation for each of the fluid phases. In addition, there will be transport equations for each solute component such as flavor components.

Variations of the continuum porous media formulation are available, the most notable one being a frontal approach to evaporation or a sharp interface phase change formulation (also called moving boundary formulation; Farid, 2002). The liquid water and water vapor transport equations can also be combined, leading to the simple diffusion equation with an effective diffusivity - perhaps the most widely used model in food process engineering. There are also phenomenological approaches (Luikov, 1975) to multiphase transport in porous media whose origin in terms of averaging have not been demonstrated and many of the transport coefficients in this model cannot be traced to standard properties. Food structures can also include two different ranges of porosities (such as inter-particle and intra-particle) and can be modeled using dual porosity models, as described by Zygalakis et al. (2011) for transport of nutrients in root hair or by Wallach et al. (2011) for flow of water during rehydration of foods.

A deforming (shrinking/swelling) porous medium is essentially handled by treating all fluxes, discussed earlier for a rigid porous medium, to be those relative to the solid matrix, and combining this with a velocity of the solid matrix that comes from deformation obtained from solid mechanical stress-strain analysis (also assuming macroscale continuum). Since the solid has a finite velocity, the mass flux of a species with respect to a stationary observer can be written as a sum of the flux with respect to solid and the flux due to movement of the solid with respect to a stationary observer (Rakesh and Datta, accepted for publication). Pressure gradients that cause deformation can originate from a number of possible mechanisms: gas pressure due to evaporation of water or gas release (as for carbon dioxide in baking); capillary pressure; or swelling pressure that are functions of the temperature and moisture content of the food material. Kelvin's law can be used to estimate capillary pressure from water activity. Flory-Rehner theory has also been used to estimate this pressure (van der Sman, 2007a). Furthermore, swelling pressure has been estimated from water holding capacity in case of meat (e.g., Dhall and Datta, 2011). The solid matrix can be treated as elastic, viscoelastic or following other material models and the corresponding strain energy function can be used with the linear momentum balance equation for the deforming solid.

\subsection{Limitations of the macroscale formulation and the need for multiscale formulation}

In the aforementioned macroscale formulations, the food is replaced by a structureless continuum. This means that its properties would not change when subdivided. Of course a food can still consist of different materials, but they all should be continuum materials and have dimensions of the same order of magnitude as the processes that are studied. The continuum hypothesis has a very important advantage: the equations of mathematical physics that describe phenomena such as heat conduction, fluid flow, water transport, diffusion of species apply, and commercial finite element or finite volume codes can be used to solve them. However, the material properties that are required are apparent properties rather than real physical constants: they implicitly depend on the fine structure of the material and need to be measured experimentally. Given the ever growing variety of foods this is simply not possible for all foods. Also, their measurement is not trivial (various ways of estimating them are summarized in Gulati and Datta, submitted for publication). This problem, however, can be alleviated using multiscale simulation.

Material properties can also be predicted using the effective medium theory of Maxwell-Garnett and its extensions (e.g., van der Sman, 2008) where the material is considered as a two-phase medium (a matrix with inclusions). Such predictions, however, have been limited in the past, perhaps since the specific microstructure of the material is generally not included. Thermodynamics-based approaches, such as the one used for predicting water activity (van der Sman and Boer, 2005), are also unlikely to be universally applicable to all types of physical properties unless such approaches can include microstructural information.

Another limitation of continuum modeling is the fact that the actual details of microscale heterogeneity, as is important in some food applications (Halder et al., 2011; Ho et al., 2011), will not be picked up by macroscale models by their very design, and microscale models would be needed.

Theoretically, a comprehensive model could be conceived that incorporates geometrical features from the macroscale to the smallest relevant scale. The size of the corresponding computational model (thus finite element mesh) would, however, surpass both the memory and computational power of current high performance computers by many orders of magnitude. Also, the continuum hypothesis breaks down at smaller scales; the particle nature of materials becomes dominant. The numerical methods to solve such problems scale even worse with size. Multiscale modeling provides an alternative paradigm for modeling processes at spatially and temporally relevant scales for food, while still accounting for microstructural features.

\section{Multiscale modeling paradigm}

Multiscale models are basically a hierarchy of sub-models which describe the material behavior at different spatial scales in such a way that the sub-models are interconnected. The principle of multiscale modeling is shown in Fig. 4. Typically, equations for the fine scale are solved to calculate apparent material properties for models that operate at a coarser scale. The up-scaling of fine scale solutions to a coarse solution is known as upscaling, homogenization or coarse-graining (Brewster and Beylkin, 1995; Mehraeen and Chen, 2006). The algorithm proceeds from scale to scale until the scale of interest is reached. The reverse method is called downscaling, localization or fine-graining and is used when local phenomena that depend on macroscale variables are required. Consider, for example, failure of fruit tissue due to compressive loading. In the homogenization step, apparent mechanical properties of the macroscopic model are derived through homogenization from numerical experiments at smaller scales. Using these apparent properties, the stress distribution inside the fruit is calculated at the macroscale. Failure is likely to occur in zones of maximal stress. Thus, in the localization step, mesoscale models will then 


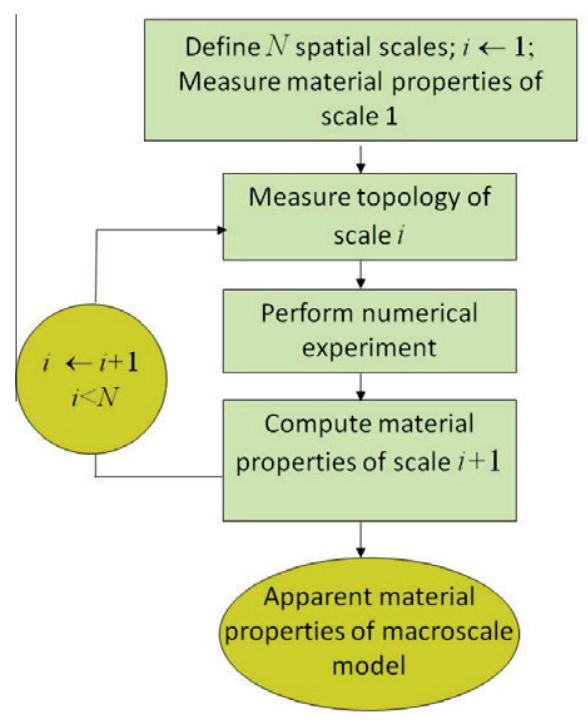

A. Homogenization

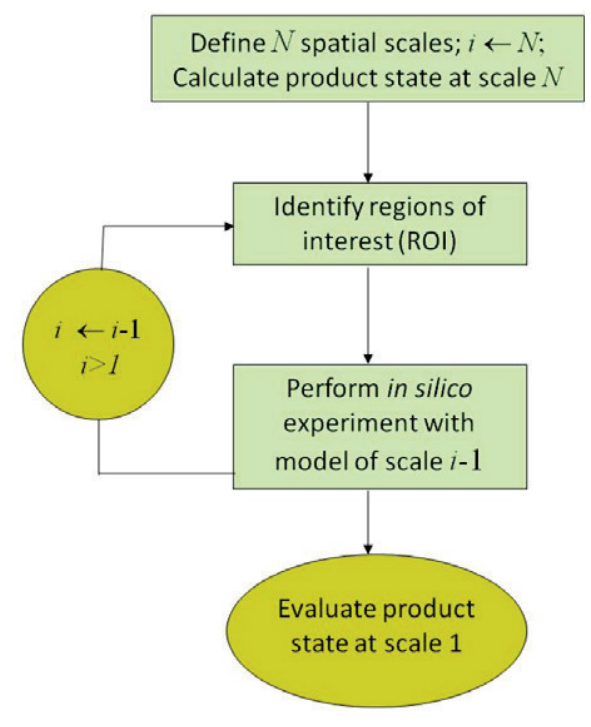

B. Localization

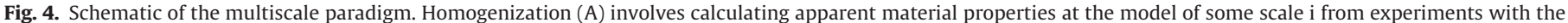

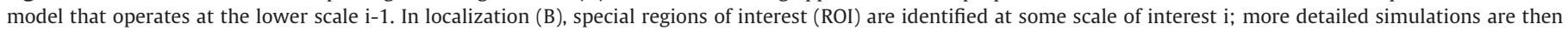
carried out in this ROI using the model that operates at scale i-1. (Adapted from Ho et al., 2011).

be used to calculate stresses on individual cells in these affected zones. Using microscale models stresses in the cell wall of these cells will be evaluated. Cell failure will occur when an appropriate failure criterion is violated, e.g., when the cell wall tensile stress exceeds the tensile strength of the cell wall.

\section{Numerical techniques for multiscale analysis}

In this section we will give an overview of the most used numerical methods for solving physics problems at different scales. A particular challenge of multiscale modeling is that at the mesoscale and beyond the physics gradually changes: fluids behave like a collection of particles, the spatial and temporal variation of macroscopic variables becomes huge, and Brownian motion may become important. For example, water transport at the microscale and up is governed by the Navier-Stokes equations that predict a parabolic velocity profile in cylindrical channels. If the diameter of the channel is of the same size as the size of the water molecule, there is too little space to fully develop a velocity profile, and the individual molecules will line up and move in an orderly pattern through the nanochannel (Mashl et al., 2003). Continuum physics based simulation methods such as the finite element and finite volume methods are no longer applicable, and meshless particle methods, Lattice Boltzmann or molecular dynamics are required.

\subsection{Finite element and finite volume method}

The finite element method is a very flexible and accurate method for solving partial differential equations (Zienkiewicz and Taylor, 2005). In this method, the continuum is subdivided in elements of variable size and shape that are interconnected in a finite number of nodal points. In every element the unknown solution is expressed as a linear combination of so-called shape functions. In a next step the equations are spatially discretized over the finite element mesh using a suitable technique such as the Galerkin weighted residual method. Hereto the residual that is obtained by substituting the approximate solution in the governing partial differential equation is orthogonalized with respect to the shape functions. Depending on whether time is an independent variable, the end result is a system of algebraic equations or ordinary differential equations; the latter is then usually discretized using a finite difference approximation. The finer the mesh, the better the approximation but also the more computational time that is required to solve the resulting equations.

The finite volume method is very popular for solving fluid transport problems and is at the basis of many commercial computational fluid dynamics codes (Hirsh, 2007). As in the finite element method, the computational domain is discretized in finite volumes. The conservation laws underlying the governing equations are imposed at the level of every finite volume, and applying Green's theorem then naturally leads to a relationship between fluxes at the finite volume boundaries. These fluxes are approximated by finite differences, and the end result is again a system of algebraic or differential equations in the unknowns at the discretization points.

\subsection{Meshless particle methods}

In many mechanical systems, grid based methods such as the finite element method are very efficient and robust for simulating continuum materials undergoing small or moderate deformations. Yet, these methods are usually less suited or may even run into trouble when problems with excessive deformations, fracturing, or free surfaces are encountered. The discrete nature of some materials requires an alternative way of calculating dynamics. The key idea in so-called meshless particle methods is that the material is mass-discretized into material points. These points are not related by a mesh. Similar to molecular dynamics simulations, they only interact through pairwise interaction potentials when their relative distance is smaller than the cutoff distance (Tijskens et al., 2003). In the discrete element method (DEM), the interaction forces are usually computed from linear spring-dashpot elements, or Hertz theory. An instructive example is the collision of apples in harvesting or transport, where the exerted forces are calculated to predict bruising volume (Van Zeebroeck et al., 2006a,b).

Yet, simulating a microscopic multi-body system of macroscopic dimensions would confront us with an unrealizable computational effort. In such cases, the discrete particles in the system need to be coarse grained and the stiff interactions are modified 
Table 1

Application areas for micro-mesoscale simulation of foods using Lattice Boltzmann.

\begin{tabular}{|c|c|}
\hline Application area & Key publications \\
\hline Emulsion flow/breakup/microfluidics & Biferale et al. (2011), Kondaraju et al. (2011), Van der Graaf et al. (2006) \\
\hline Pickering emulsion & Jansen and Harting (2011) \\
\hline Surfactant + Droplet & Farhat et al. (2011), Liu and Zhang (2010); Van der Sman and Van der Graaf (2006) \\
\hline Particle suspensions flow & Kromkamp et al. (2005); Ladd and Verberg (2001); Vollebregt et al. (2010) \\
\hline Single phase porous media flow & Sholokhova et al. (2009) \\
\hline Two-phase porous media flow & Porter et al. (2009) \\
\hline Foaming & Körner (2008) \\
\hline Digestion & Connington et al. (2009), Wang et al. (2010) \\
\hline Extruder flow & Buick (2009) \\
\hline Biofouling (membranes) & von der Schulenburg et al. (2009) \\
\hline
\end{tabular}

to softer potentials to reduce the number of particles. In the last 20 years, there has been an increasing interest of smooth particle applied mechanics (SPAM). In SPAM, the particle interactions are basically derived from a continuum law by smearing out variables associated with a particle to neighboring particles (within cutoff distance). This is done by a "kernel" interpolant. Any set of PDEs can be transformed into a set of ODEs without the need for a mesh or remeshing. This method thus combines the discrete nature of materials with its continuum properties and is thus well suited for systems undergoing large deformations with cracking. Notorious examples of this method are abundant in fluid dynamics, known as Smoothed Particle Hydrodynamics (SPH) (Monaghan, 2011). More recent applications can be found in soil mechanics (Bui et al., 2007) and soft tissue (Hieber and Komoutsakos, 2008). Other meshless methods include Brownian dynamics. Guidelines about which method should be used at a particular spatial scale were given by van der Sman (2010).

\subsection{The Lattice Boltzmann method}

The Lattice Boltzmann method is most suitable for microscale and mesoscale simulations, and has found significantly more applications in food science than any other mesoscale method (van der Sman, 2007b). In the Lattice Boltzmann method, materials and fluids are represented as quasi-particles populating a regular lattice. They interact via collisions, which adhere the basic conservation laws of mass, momentum and energy. The collision rules follow a discretized version of the Boltzmann equation, which also governs the collisions of particles on the molecular level. In Lattice Boltzmann the particles do not represent individual molecules, but parcels of fluid. The grid spacing can be of similar order as in traditional macroscale methods as the finite element or finite volume method. It is the discretization of space, time and momentum what makes Lattice Boltzmann different from the traditional method. The method can handle complex bounding geometries with simple bounceback rules of the particles, which can easily be generalized to moving boundaries - as is required for modeling particle suspension flow (Ladd and Verberg, 2001). Its connection to kinetic theory via the Boltzmann equation makes it straightforward to link it to thermodynamic theories, describing the driving force of transport processes (Swift et al., 1996; van der Sman, 2006). These last two properties make the Lattice Boltzmann a versatile vehicle for doing mesoscale simulations of dispersions. In a multiscale simulation framework for food processing the Lattice Boltzmann can be used as a solver at the mesoscale, or at the macroscale for flow problems through complicated geometries like porous media. To give an impression of the versatility, references to several applications that are relevant from the food perspective are summarized in Table 1.

\subsection{Molecular dynamics}

Molecular dynamics is used to study the behavior of materials at the molecular scale (Haile, 1997). In molecular dynamics the movement of molecules is computed by solving Newton's equation of motion using time steps of the order of 1 femtosecond $\left(10^{-15} \mathrm{~s}\right)$. The forces between the molecules are computed from the potential field that is caused by covalent bonds and long range van der Waals and electrostatic interactions. The van der Waals term is often modeled with a Lennard-Jones potential, the electrostatic term with Coulomb's law. The evaluation of these potentials is computationally the most intensive step of a molecular dynamics simulation. Molecular dynamics can be considered as a discrete element method. In food science, molecular dynamics is hardly applied (Limbach and Kremer, 2006), with the exception of the studies by Limbach and Ubbink (2008) and by Brady and coworkers (Lelong et al., 2009).

\section{Homogenization and localization}

Coupling of models at fine and coarse scales is an essential feature of multiscale methods. We will focus here on problems where there is spatial scale separation - the length scale of the heterogeneities of the microscale is small compared to the dimensions of the macroscale; in this case the multiscale paradigm is most effective in terms of reducing computational time compared to a macroscopic model that is numerically resolved to the microscale. We will not discuss the classical volume averaging approach such as used by Bear (1972) and Whitaker (1977) in which the homogenization is an essential part of the construction of the continuum equations and that has been propagated for years for food engineering applications by Datta's group (e.g., Ni and Datta, 1999; Ni et al., 1999).

The original mathematical homogenization procedure involves applying a second order perturbation to the governing equation. When applied to a diffusion equation the result is a homogenized diffusion equation incorporating an apparent diffusivity that can be calculated by solving yet another diffusion equation called the cell equation (Pavliotis and Stuart, 2008). Usually a more pragmatic approach is taken, and the apparent diffusivity is calculated by solving the microscale model with appropriate boundary conditions on a microscopic computational domain. When the microscale model is a partial differential equation, often periodic boundary conditions are applied. The selection of boundary conditions is much more complicated when the microscale model is a discrete model (E et al., 2007). This method is also known as sequential (serial) coupling (Ingram et al., 2004), as the computation of the apparent material properties can be considered as a preprocessing step that can be done independent from the solution of the macroscale model.

Sequential coupling requires that some assumptions need to be made about the constitutive equations, such as for a diffusion process the relationship between flux and concentration (or potential) gradients. This approach is valid as long as the constitutive equation depends only on a limited number of variables. When the constitutive relation depends on many variables, sequential coupling 
is difficult and the heterogeneous multiscale method (HMM) is more appropriate. This method is particularly suited for linking submodels of different nature - e.g., a continuum model at the macroscale and a discrete element model at the microscale (E et al., 2007). The starting point is usually a finite element or finite volume discretization of the macroscale equation. The element wise construction of the finite element matrices involves the numerical integration of an expression incorporating local fluxes or other variables that are a function of the microstructure. The HMM exploits the fact that these variables are only required in the (few) numerical integration points. The microscale model is, therefore, solved numerically in a small domain surrounding these integration points. The HMM thus does not explicitly compute a homogenized value of the material properties. The HMM is a top-down method: it starts at the macroscale and calculates the local information it needs using the microscale model (localization or downscaling), where initial and boundary conditions are set by the macroscale model. It is an example of concurrent (or parallel) coupling, as the microscale and the macroscale model are simultaneously solved, and it is equation-free - no assumptions regarding the constitutive equations need to be made. An alternative method involves the computation of shape functions for use at the macroscale, based on the solution of a microscale problem in every element (Nassehi and Parvazinia, 2011). For the latter, a different set of shape functions called 'bubble' functions are used. This method is a bottom-up method as it starts from the microscale. For further details the reader is referred to the literature.

Localization is the inverse of homogenization and has received far less attention in the food literature. The approach outlined in Fig. 4b can be applied once the macroscale solution is known. One simply zooms in on the area of interest, e.g., often where the smallest or largest values of the variable of interest or its gradient are expected, and uses the microscale model to investigate what happens at the microscale.

\section{Applications}

Multiscale modeling is a relatively new area in food engineering, and the literature is relatively scarce. We will discuss a few representative publications, mostly from the authors of this article.

Multiscale modeling using serial coupling has been applied to postharvest storage of fruit and vegetables by Nicolaï and coworkers. An early application was presented by Veraverbeke et al. (2003a,b) who used microscale models for water transport through different microscopic surface structures in apple skin, such as cracks in the epicuticular wax layer and closed and open lenticels, to compute an apparent water diffusion coefficient for the entire cuticle. The latter was incorporated in a macroscopic water transport model that was used to evaluate the effect of storage conditions on water loss. Ho et al. (2009, 2010a, 2011) developed a multiscale model to describe metabolic gas exchange in pear fruit during controlled atmosphere storage. The microscale gas exchange model included equations for the transport of respiratory gasses in the intercellular space and through the cell wall and plasmalemma into the cytoplasm, and incorporated the actual tissue microstructure as obtained from synchrotron radiation tomography images (Verboven et al., 2008). Cellular respiration was modeled as well. The macroscale gas transport model included diffusion, permeation and respiration. The model was validated (Ho et al., 2010b) and used to study hypoxia in fruit during storage. An example of multiscale modeling at larger spatial scales in postharvest applications was given by Delele et al. (2008, 2009). They investigated high pressure fogging systems to humidify controlled atmosphere storage rooms using a CFD based multiscale model. At the fine scale, the flow through stacked products in boxes was predicted using a combination of discrete element and CFD modeling. At the coarse scale, a CFD model for a loaded cool room was developed to predict the storage room air velocity, temperature and humidity distributions and fate of the water droplets. The loaded product was modeled as a porous medium, and the corresponding anisotropic loss coefficients were determined from the fine scale model. A Lagrangian particle tracking multiphase flow model was used for simulating droplet trajectories. Recently, a new computational multiscale paradigm based on SPH-DEM particle simulations, computational homogenization, and a finite element formulation has been developed and applied for calculating mechanical properties such as the intracellular viscosity and the cell wall stiffness, and the dynamic tissue behavior, including bruising, of fruit parenchyma tissue (Ghysels et al., 2009; Van Liedekerke et al., 2011).

For particle suspensions, representing beverages like milk and beer, van der Sman and coworkers have developed a multiscalesimulation approach, using Lattice Boltzmann at the meso, micro and macroscale (van der Sman, 2009). The levels differ in the resolution of the particle size with respect to the computational grid. The three levels are serially coupled, and fine-scale simulations render closure relations for the coarser scale, such as the particle friction coefficient and particle stress (osmotic pressure). These closures are used in a mixture model (Vollebregt et al., 2010) describing shear-induced migration of food suspensions in fractionation applications such as beer microfiltration (van der Sman et al., 2012). Similar closure relations are derived for particle suspensions confined in microfluidic devices (van der Sman, 2010, 2012 ), i.e. deterministic ratchets designed for fractionation of food suspensions (Kulrattanarak et al., 2011).

Furthermore, the van der Sman group recently implemented a serially coupled multiscale model (Esveld et al., 2012a,b), which predicts the dynamics of moisture diffusion into cellular solid foods, following their earlier proposal for the multiscale framework for food structuring (van der Sman and Van der Goot, 2008). They determined the characteristics of the air pores and their connectivity through 3-D image analysis of X-ray micro CT images and used this information to construct a discrete microscale network model. The model accounted for local diffusive vapor transport through the pores and moisture sorption in the lamellae. The characteristics of the network were volume averaged to a steady state vapor conductivity and a quasi-steady-state sorption time constant. These parameters were incorporated into a macroscale model consisting of two coupled differential equations. The authors successfully predicted experimental dynamical moisture profiles of crackers with a fine and coarse morphology measured by means of MRI.

Guessasma et al. $(2008,2011)$ presented a multiscale model for mechanical properties of bakery products. They considered both an artificial foam generated by means of the random sequential addition algorithm as well as X-ray micotomography images. The overall elastic modulus was computed by assuming linear elastic properties of the solid phase, and a fair agreement with measured values was found.

\section{Future prospects}

Multiscale modeling of food processes is still at its infancy, and there are many problems to be solved yet.

\subsection{Scale separation}

Classical multiscale simulation methods, based on homogenization and/or localization, implicitly assume separations of time and length scales. If the size of the representative elementary volume 
at the fine scale is of the same order of magnitude as the characteristic length of the coarse scale then the scales are not separated and serial coupling is not possible. Whether this is relevant in food materials and, if so, the numerical consequences it causes remain to be investigated.

\subsection{Homogenization methods}

Coupling the different scales is not trivial. In most applications so far homogenization has been done through numerical experiments using serial coupling. Typically, boundary conditions that mimic the conditions of the actual experiment are applied - often a Dirichlet boundary condition in one direction and a zero flux Neumann boundary condition in the other direction; however, these boundary conditions are artificial and are only there because the computational domain needs to be truncated and localized. Yue and E (2007) found that the best results for elliptic problems are obtained with periodic boundary conditions. To date it is also still not possible to couple directly the nanoscale to the macroscale of the food product. In foods the micro/mesoscale level is very important, because this is the length scale of the dispersed phases which determine the food structure/texture. At this length scale the physics of foods is very rich, but quite unexplored (Donald, 1994; Mezzenga et al., 2005; Ubbink et al., 2008; van der Sman and Van der Goot, 2008). Only since two decades, computational physicists have been able to simulate this intermediate level thanks to the development of mesoscale simulation techniques (Chen and Doolen, 1998; Groot and Warren, 1997). For food applications it has been rarely used, except for the Lattice Boltzmann method, which has been used by van der Sman and coworkers (Kromkamp et al., 2005; van der Graaf et al., 2006; van der Sman, 1999, 2007b, 2009; van der Sman and Ernst, 2000), and the Dissipative Particle Dynamics method, which has been used by Dickinson and coworkers (Whittle and Dickinson, 2001) and by Groot and coworkers (Groot, 2003, 2004; Groot and Stoyanov, 2010). The main hurdle for the development of mesoscale simulation methods is to bridge the continuum (Eulerian) description of the fluid dynamics with the particulate (Lagrangian) description of the dispersed phases. The Lattice Boltzmann method has shown to be particular successful in this respect, viewing the thousands of citations of the method in the ISI database.

Parallel multiscale methods are also thought to be very useful for food science, albeit that full blown parallel micro-macro multiscale simulations like the HMM method (E et at., 2007) are computationally challenging to implement. We believe that such simulations are particular useful for applications involving the structuring of foods via phase transitions as occurs during intensive heating (frying, baking, puffing) or freezing. Such a multiscale model has been developed already quite early (Alavi et al., 2003), to describe bubble formation in extruded starchy foods.

\subsection{Statistical considerations}

The selection of the computational domain in the serial method is very important. As outlined before, statistical techniques can be used to calculate the size of the representative elementary volume that can be used as the computational domain. However, the structural heterogeneity is not necessarily stationary and may vary within the computational domain of the coarse model. It is important to repeat calculations of apparent material properties on several geometrical models of the fine scale and analyze them statistically (see Ho et al., 2011, for an example).

In many applications the structure of the fine scale is in fact random; for example, apple parenchyma cells have random shapes and dimensions. In view of serial upscaling methods, this implies that the corresponding apparent material property is a random field - a quantity that fluctuates randomly in space. In this case stochastic finite element methods can be used to compute the propagation of these random fluctuations through the governing equation. Perturbation methods have been used as a cheap alternative to Monte Carlo simulations; they can be considered as a stochastic equivalent of formal mathematical averaging and homogenization methods (Pavliotis and Stuart, 2008). Applications in food engineering have been described by Nicolaï et al. (1997, 1998, 2000 and Scheerlinck et al. (2000). The relationship between random structure at the fine scale and random apparent properties has not been investigated yet, and more research is required.

\subsection{Required resolution}

A fundamental question about multiscale modeling is how deep we have to dive into the multiscale structure of the food material. This depends on the answers we seek. If we use multiscale modeling to predict food parameters, the finest level we need to resolve is that where the material properties become physical properties that are sufficiently generic, available in the literature, or easily measureable. However, as our understanding of the fine structure of food materials is ever increasing, the required resolution of the multiscale model is also likely to increase. For example, a model for water transport in apple would incorporate at the nanoscale the permeability of the phosopholipid bilayer membrane of the cell. However, membranes contain specialized proteins, called aquaporins, to facilitate water transport; not only are there different types of aquaporins, their density in the membrane is also variable. So, either we need to measure the permeability of the particular membranes we are interested in, or we need to compute water transport during the aquaporins using molecular dynamics techniques. Unfortunately, measurements of physical properties and geometrical features become increasingly more difficult at smaller scales. Also, the smaller the scale, the more features will likely affect the processes that are investigated. Clearly, the finest scale that one chooses to model will always be a compromise between accuracy and complexity; understanding food processes will require a finer resolution than the computation of material properties.

\subsection{Food structuring processes}

The emphasis of this review has been on predicting food material properties. But an equally important potential application of multiscale simulation is for the prediction of food structuring or texturing processes (van der Sman and Van der Goot, 2008). During these processes one manipulates or creates dispersed phases, frequently via phase transitions like boiling or freezing as in baking. This process requires a description of the evolution of the dispersed phase at the meso/microscale. The structuring process is driven by applied external fields, like temperature and moisture gradients, or shearing flows. Hence, this requires a parallel/concurrent coupling between the macroscale and micro/mesoscale. Note that this coupling is two-way, the dispersed phases evolve to the local value of the macroscopic fields, but they can change material properties like porosity and thus thermal conductivity - which changes the penetration of the applied external fields into the food. One example of such a multiscale model is by Alavi et al. (2003), describing the expansion of a food snack, where the evolution of a bubble is described by a cell model. A similar model was applied recently (van der Sman and Broeze, 2011) to indirectly expanded snacks - where a proper thermodynamic description of the phase transitions of starch was used (van der Sman and Meinders, 2010).

Advancement in this field can be quite hindered by the lack of knowledge of the physics at the mesoscale, which requires proper 
coupling of thermodynamics to transport processes like flow, heat and mass transfer at the mesoscale. An example of such a coupling is shown by van der Sman and van der Graaf (2006) for a surfactant stabilized emulsion droplet. In real foods the stabilization of dispersed phases is done by a mixture of components from a large collection of phospholipids, particulates, fat crystals, proteins and surfactants. One can imagine the challenge we face in the physics at the mesoscale.

\subsection{Food process design and control}

Multiscale models by their very nature can potentially provide a more accurate description of how foods change during processing operations. It is, therefore, reasonable to expect that they will be used increasingly for food process design purposes to manipulate food quality attributes at a much better spatial resolution than currently possible. The much higher computational burden, though, has limited the use of multiscale models for food process design so far. This is even more so in process control applications where typically models of limited complexity are required. In this case formal model reduction techniques such as Galerkin projection methods (Balsa-Canto et al., 2004) could be applied to obtain a model of reduced complexity suitable for controller design. Examples yet have to appear in the literature.

\section{Conclusions}

Multiscale modeling is a new paradigm for analyzing and designing food processes. Its main advantage is that it can be used for calculating material properties of foods - one of the major hurdles that prevent widespread use of modeling in food process design and engineering, but also to establish constitutive equations. It also provides means to understand how food properties at the macroscale are affected through processing by properties and geometrical features at the microscale and beyond, but also enables to translate macroscale behavior into changes happening at the microscale. Once such relationships are known, they can be used for food structural engineering - designing the food at the microscale so that it has desirable functional and quality attributes at the macroscale (Aguilera, 2005; Guessasma et al., 2011). In other fields of research such as materials engineering, multiscale modeling is becoming a mainstream methodology for tailoring or customizing the microstructure of materials to obtain specific properties (e.g., Ghosh and Dimiduk, 2010; Kenney and Karan, 2007). Perspectives for foods applications are given by Aguilera (2005) and include aerating foams, both solid (e.g., bread) and liquid (e.g., whipped cream); entrapment of water droplets in food products, e.g. for mayonnaises or processed cheese (Heertje et al., 1999); and molecular gastronomy. The main hurdle seems to be our lack of understanding of the physics of foods at the microscale and beyond, and more research is definitely required in this area.

\section{Uncited references}

Chen and Doolen (1998), Nguyen et al. (2006), Nicolaï and De Baerdemaeker (1997), Nicolaï et al. (2000,1998), Seo et al. (2010) and Tanikawa and Shimamoto (2009)).

\section{Acknowledgements}

We would like to thank the Flanders Fund for Scientific Research (FWO Vlaanderen, project G.0603.08, project G.A108.10N), the KU Leuven (project OT/08/023) and the EU (project InsideFood FP7-226783) for financial support. The opinions expressed in this document do by no means reflect their official opinion or that of its representatives. Thijs Defraeye and Quang Tri Ho are postdoctoral fellows of the Research Foundation - Flanders (FWO) and acknowledge its support.

\section{References}

Adedeji, A.A., Ngadi, M.O., 2011. Microstructural characterization of deep-fat fried breaded chicken nuggets using x-ray micro-computed tomography. Journal of Food Process Engineering 34 (6), 2205-2219.

Aguilera, J.M., 2005. Why food microstructure? Journal of Food Engineering 67 (12), 3-11.

Alavi, S.H., Rizvi, S.S.H., Harriot, P., 2003. Process dynamics of starch-based microcellular foams produced by supercritical fluid extrusion. I: model development. Food Research International 36 (4), 309-319.

Anonymous (2012). Merriam-Webster online. Available from <http:// www.merriam-webster.com/>. Accessed 26 January 2012.

Ball, C.O., 1923. Thermal process time for canned food. Bulletin of the National Research Council 7 (Part I), No. 37.

Balsa-Canto, E., Alonso, A.A., Banga, J.R., 2004. Reduced-order models for nonlinear distributed process systems and their application in dynamic optimization Industrial \& Engineering Chemistry Research 43, 3353-3363.

Bear, J., 1972. Dynamics of Fluids in Porous Media, first ed. American Elsevier Publishing Company Inc., New York.

Becker, B.R., Fricke, B.A., 1999. Food thermophysical property models. International Communications in Heat and Mass Transfer 26 (5), 627-636.

Biferale, L., Perlekar, P., Sbragaglia, M., Srivastava, S., \& Toschi, F. (2011). A Lattice Boltzmann method for turbulent emulsions, Journal of Physics: Conference Series, 318(5), art. nr. 052017 (10pp.).

Brewster, M.E., Beylkin, G., 1995. A multiresolution strategy for numerical homogenization. Applied and Computational Harmonic Analysis 2, 327-349.

Bui, H.H., Fukagawa, R., Sako, K., Ohno, S., 2007. Lagrangian mesh-free particles method (SPH) for large deformation and failure flows of geomaterial using elastic-plastic soil constitutive model. International Journal for Numerical and Analytical Methods in Geomechanics 32 (12), 1537-1570.

Centonze, V., Pawley, J.B., 2006. Tutorial on practical confocal microscopy and the use of the confocal test specimen. In: Pawley, J.B. (Ed.), Handbook of Biological Confocal Microscopy, third ed. Springer Science+Business Media, LLC, pp. 627647 (Chapter 35).

Chen, S.Y., Doolen, G.D., 1998. Lattice Boltzmann method for fluid flows. Annual Review of Fluid Mechanics 30 (1), 329-364.

Collewet, G., Bogner, P., Allen, P., Busk, H., Dobrowolski, A., Olsen, E., Davenel, A. 2005. Determination of the lean meat percentage of pig carcasses using magnetic resonance imaging. Meat Science 70, 563-572.

Connington, K., Kang, Q., Viswanathan, H., Abdel-Fattah, A., Chen, S., 2009 Peristaltic particle transport using the lattice Boltzmann method. Physics of Fluids 21 (5), art. nr. 053301 (16pp.)

Datta, A.K., 2007a. Porous media approaches to studying simultaneous heat and mass transfer in food processes. I: problem formulations. Journal of Food Engineering 80 (1), 80-95.

Datta, A.K., 2007b. Porous media approaches to studying simultaneous heat and mass transfer in food processes. II: property data and representative results. Journal of Food Engineering 80 (1), 96-110.

Datta, A.K., 2008. Status of physics-based models in the design of food products, processes, and equipment. Comprehensive Reviews in Food Science and Food Safety 7 (1), 121-129.

Delele, M., Tijskens, E., Atalay, Y., Ho, Q., Ramon, H., Nicolaï, B., Verboven, P., 2008. Combined discrete element and CFD modeling of airflow through random stacking of horticultural products in vented boxes. Journal of food engineering 89 (1) 33-41.

Delele, M.A., Schenk, A., Tijskens, E., Ramon, H., Nicolaï, B.M., Verboven, P., 2009. Optimization of the humidification of cold stores by pressurized water atomizers based on a multiscale CFD model. Journal of Food Engineering 91 (2), 228-239.

Dhall, A., Datta, A.K., 2011. Transport in deformable food materials: a poromechanics approach. Chemical Engineering Science 66 (24), 6482-6497.

Donald, A.M., 1994. Physics of foodstuffs. Reports on Progress in Physics 57 (11), 1081-1135.

E, W., Engquist, B., Li, X.T., Ren, W.Q., Vanden-Eijnden, E., 2007. Heterogeneous multiscale methods: a review. Communications in Computational Physics 2 (3), 367-450.

Esveld, D.C., van der Sman, R.G.M., van Dalen, G., van Duynhoven, J.P.M., Meinders, M.B.J., 2012a. Effect of morphology on water sorption in cellular solid foods. Part I: pore scale network model. Journal of Food Engineering 109 (2), 301-310.

Esveld, D.C., van der Sman, R.G.M., Witek, M.M., Windt, C.W., van As, H., van Duynhoven, J.P.M., Meinders, M.B.J., 2012b. Effect of morphology on water sorption in cellular solid foods. Part II: Sorption in cereal crackers. Journal of Food Engineering 109 (2), 311-320.

Falcone, P.M., Baiano, A., Zanini, F., Mancini, L., Tromba, G., Montanari, F., Del Nobile, M.A., 2004. A novel approach to the study of bread porous structure: phasecontrast X-ray micro-tomography. Journal of Food Science 69 (1), 38-43.

Falcone, P.M., Baiano, A., Conte, A., Mancini, L., Tromba, G., Zanini, F., Del Nobile, M.A., 2006. Imaging techniques for the study of food microstructure: a review. Advances in Food \& Nutrition Research 51, 205-263.
1042

1043

1044

1045

1046

1047

1048

1049

1050

1051

1052

1053

1054

1056

1057

1058

1059

1060

1061

1062

1063

1064

1065

1066

1067

1068

1069

1070

1071
1072

073

1073
1074

1075

1076

1077

1078

1079

1080

1081

1082

1083

1084

1085

1086

1087

1088
1089

1090

1091

1092

1093

1094

1095

1096

1097

1098
1099

1100

1101

1102

1103

1104

1105

1106

1107

1108

1109

1111

1112

1113

1114

1115

1116

1117
1118 
Farhat, H., Celiker, F., Singh, T., Lee, J.S., 2011. A hybrid lattice Boltzmann model for surfactant-covered droplets. Soft Matter 7, 1968-1985.

Farid, M., 2002. The moving boundary problems from melting and freezing to drying and frying of food. Chemical Engineering and Processing 41 (1), 1-10.

Fikiin, K.A., Fikiin, A.G., 1999. Predictive equations for thermophysical properties and enthalpy during cooling and freezing of food materials. Journal of Food Engineering 40, 1-6.

Frisullo, P., Laverse, J., Marino, R., Del Nobile, M.A., 2009. X-ray computer tomography to study processed meat micro-structure. Journal of Food Engineering 94, 283-289.

Frisullo, P., Conte, A., Del Nobile, M.A., 2010. A novel approach to study biscuits and breadsticks using X-Ray computed tomography. Journal of Food Science 75 (6), E353-358.

Frisullo, P., Laverse, J., Barnabà, M., Navarini, L., Del Nobile, M.A., 2012. Coffee beans microstructural changes induced by cultivation processing: an X-ray microtomographic investigation. Journal of Food Engineering 109 (1), 175-181.

Ghosh, S., Dimiduk, D., 2010. Computational Methods for Microstructure-Property Relationships, first ed. Springer, New York.

Ghysels, P., Samaey, G., Tijskens, E., Van Liedekerke, P., Ramon, H., Roose, D., 2009. Multi-scale simulation of plant tissue deformation using a model for individual cell mechanics. Physical Biology 6 (1), art. nr. 016009 (14pp.).

Groot, R., 2003. Electrostatic interactions in dissipative particle dynamicssimulation of polyelectrolytes and anionic surfactants. The Journal of Chemical Physics 118 (24), art. nr. 11265 (13pp.).

Groot, R., 2004. Applications of dissipative particle dynamics. Novel Methods in Soft Matter Simulations - Lecture Notes in Physics 640, 5-38.

Groot, R.D., Stoyanov, S.D., 2010. Equation of state of surface-adsorbing colloids. Soft Matter 6 (8), 1682-1692.

Groot, R.D., Warren, P.B., 1997. Dissipative particle dynamics: bridging the gap between atomistic and mesoscopic simulation. Journal of Chemical Physics 107 (11), 4423-4435.

Guessasma, S., Babin, P., Della Valle, G., Dendievel, R., 2008. Relating cellular structure of open solid food foams to their Young's modulus: finite element calculation. International Journal of Solids Structure 45, 2881-2896.

Guessasma, S., Chaunier, L., Della Valle, G., Lourdin, D., 2011. Mechanical modeling of cereal solid foods. Trends in Food Science \& Technology 22, 142-153.

Gulati, T., Datta, A.K., submitted for publication. Food property prediction equations for enabling computer-aided food process engineering. Journal of Food Engineering..

Haile, J.M., 1997. Molecular Dynamics Simulation: Elementary Methods, first ed. Wiley-Interscience, New York.

Halder, A., Dhall, A., Datta, A.K., 2007. An improved, easily implementable, porous media based model for deep-fat frying. Part I: problem formulation and input parameters. Transactions of the Institution of Chemical Engineers, Part C Food and Bioproducts Processing 85 (3), 209-219.

Halder, A., Datta, A.K., Spanswick, R.M., 2011. Water transport in cellular tissues during thermal processing. American Institute of Chemical Engineers Journal 57 (9), 2574-2588.

Heertje, I., Roijers, E.C., Hendrickx, H.A.C., 1999. Liquid crystalline phases in the structuring of food products. Lebensmittel-Wissenschaft und-Technologie 31, 387-396.

Hieber, S.E., Komoutsakos, P., 2008. A Lagrangian particle method for simulation of linear and nonlinear models of soft tissue. Journal of Computational Physics 227, 9195-9215.

Hills, B., 1995. Food processing: an MRI perspective. Trends in Food Science \& Technology 6, 111-117.

Hirakimoto, A., 2001. Microfocus X-ray computed tomography and its industrial applications. Analytical Sciences 17, 123-125.

Hirsh, Ch., 2007. Numerical Computation of Internal and External Flows: The Fundamentals of Computational Fluid Dynamics, second ed. ButterworthHeinemann, Oxford.

Ho, Q., Verboven, P., Verlinden, B., Lammertyn, J., Vandewalle, S., Nicolaï, B., 2008. A continuum model for metabolic gas exchange in pear fruit. PLoS Computational Biology 4 (3), e1000023 (13pp.).

Ho, Q., Verboven, P., Mebatsion, H., Verlinden, B., Vandewalle, S., Nicolaï, B., 2009. Microscale mechanisms of gas exchange in fruit tissue. The New Phytologist $182(1), 163-174$

Ho, Q., Verboven, P., Verlinden, B., Nicolai, B., 2010a. A model for gas transport in pear fruit at multiple scales. Journal of Experimental Botany 61 (8), 2071-2081.

Ho, Q., Verboven, P., Verlinden, B., Schenk, A., Delele, M., Rolletschek, H. Vercammen, J., Nicolai, B., 2010b. Genotype effects on internal gas gradients in apple fruit. Journal of Experimental Botany 61 (10), 2745-2755.

Ho, Q., Verboven, P., Verlinden, B., Herremans, E., Wevers, M., Carmeliet, J., Nicolai, B., 2011. A 3-D multiscale model for gas exchange in fruit. Plant Physiology 155 (3), 1158-1168

Hoang, M., Verboven, P., Baelmans, M., Nicolaï, B., 2003. A continuum model for airflow, heat and mass transfer in bulk of chicory roots. Transactions of the ASAE 46 (6), 1603-1611.

Ingram, G., Cameron, I., Hangoset, K.M., 2004. Classification and analysis of integrating frameworks in multiscale modelling. Chemical Engineering Science 59 (11), 2171-2187.

Jansen, F., Harting, J., 2011. From bijels to Pickering emulsions: a lattice Boltzmann study. Physical Review E 83 (4), art. nr. 046707 (11pp.).

Keehm, Y., Mukerji, T., Nur, A., 2004. Permeability prediction from thin sections: 3D reconstruction and Lattice-Boltzmann flow simulation. Geophysical Research Letters $31(4), 1-4$.
Kenney, B., Karan, K., 2007. Engineering of microstructure and design of a planar porous composite SOFC cathode: a numerical analysis. Solid State Ionics 178 (34), 297-306.

Kondaraju, S., Farhat, H., Lee, J.S., 2011. Study of aggregational characteristics of emulsions on their rheological properties using the lattice Boltzmann approach. Soft Matter 8 (5), 1374-1384.

Körner, C., 2008. Foam formation mechanisms in particle suspensions applied to metal foams. Materials Science and Engineering: A 495 (1-2), 227-235.

Kromkamp, J., Van Den Ende, D.T.M., Kandhai, D., van der Sman, R.G.M.,,Boom, R.M., 2005 Shear-induced self-diffusion and microstructure in non-Brownian suspensions at non-zero Reynolds numbers. Journal of Fluid Mechanics 529, 253-278.

Kulrattanarak, T., van der Sman, R.G.M., Schroën, C.G.P.H., Boom, R.M., 2011. Analysis of mixed motion in deterministic ratchets via experiment and particle simulation. Microfluidics and Nanofluidics 10, 843-853.

Ladd, A., Verberg, R., 2001. Lattice-Boltzmann simulations of particle-fluid suspensions. Journal of Statistical Physics 104 (5), 1191-1251.

Lammertyn, J. Dresselaers, T., Van Hecke, P. Jancsók, P. Wevers, M., Nicolaï, B., 2003. MRI and X-ray CT study of spatial distribution of core breakdown in 'Conference' pears. Magnetic Resonance Imaging 21 (7), 805-815.

Lamnatou, Chr., Papanicolaou, E., Belessiotis, V., Kyriakis, N., 2010. Finite-volume modelling of heat and mass transfer during convective drying of porous bodies - Non-conjugate and conjugate formulations involving the aerodynamic effects. Renewable Energy 35 (7), 1391-1402.

Larabell, C.A., Nugent, K.A., 2010. Imaging cellular architecture with X-rays. Current Opinion in Structural Biology 20 (5), 623-631.

Lelong, G., Howells, W.S., Brady, J.W., Talón, C., Price, D.L., Saboungi, M.-L., 2009. Translational and rotational dynamics of monosaccharide solutions. The Journal of Physical Chemistry B 113 (39), 13079-13085.

Lim, K.S., Barigou, M., 2004. X-ray micro-tomography of cellular food products. Food Research International 37, 1001-1012.

Limbach, H.J., Kremer, K., 2006. Multi-scale modelling of polymers: perspectives for food materials. Trends in Food Science \& Technology 17 (5), 215-219.

Limbach, H.J., Ubbink, J., 2008. Structure and dynamics of maltooligomer-water solutions and glasses. Soft Matter 4 (9), 1887-1898.

Liu, H., Zhang, Y., 2010. Phase-field modeling droplet dynamics with soluble surfactants. Journal of Computational Physics 229 (24), 9166-9187.

Luikov, A.V., 1975. Systems of differential equations of heat and mass transfer in capillary-porous bodies (review). International Journal of Heat and Mass Transfer 18 (1), 1-14.

Mashl, R.J., Joseph, S., Aluru, N.R., Jakobsson, E., 2003. Anomalously immobilized water: a new water phase induced by confinement in nanotubes. Nano Letters 3 (5), 589-592.

Mebatsion, H., Verboven, P., Ho, Q., Verlinden, B., Nicolaï, B., 2008. Modeling fruit (micro) structures, why and how? Trends in Food Science \& Technology 19 (2), 59-66.

Meglinski, I.V., Buranachai, C., Terry, L.A., 2010. Plant photonics: application of optical coherence tomography to monitor defects and rots in onion. Laser Physics Letters 7 (4), 307-310.

Mehraeen, S., Chen, J.S., 2006. Wavelet Galerkin method in multi-scale homogenization of heterogeneous media. International Journal for Numerical Methods in Engineering 66, 381-403.

Mendoza, F., Verboven, P., Mebatsion, H., Kerckhofs, G., Wevers, M., Nicolaï, B., 2007. Three-dimensional pore space quantification of apple tissue using X-ray computed microtomography. Planta 226 (3), 559-570.

Mezzenga, R., Schurtenberger, P., Burbidge, A., Michel, M., 2005. Understanding foods as soft materials. Nature Materials 4 (10), 729-740.

Monaghan, J.J., 2011. Smoothed particle hydrodynamics and its diverse applications. Annual Reviews of Fluid Mechanics 44, 323-346.

Moreno-Atanasio, R., Williams, R.A., Jia, X., 2010. Combining X-ray microtomography with computer simulation for analysis of granular and porous materials. Particuology 8 (2), 81-99.

Nahor, H., Hoang, M., Verboven, P., Baelmans, M., Nicolaï, B., 2005. CFD model of the airflow, heat and mass transfer in cool stores. International Journal of Refrigeration 28 (3), 368-380

Nassehi, V., Parvazinia, M., 2011. Finite Element Modeling of Multiscale Transport Phenomena, first ed. Imperial College Press, London.

Nguyen, T., Dresselaers, T., Verboven, P., D'hallewin, G., Culeddu, N., Van Hecke, P. Nicolaï, B., 2006. Finite element modelling and MRI validation of 3D transient water profiles in pears during postharvest storage. Journal of the Science of Food and Agriculture 86 (5), 745-756.

$\mathrm{Ni}$, H., Datta, A.K., 1999. Heat and moisture transfer in baking of potato slabs. Drying Technology 17, 2069-2092.

Ni, H., Datta, A.K., Torrance, K.E., 1999. Moisture transport in intensive microwave heating of wet materials: a multiphase porous media model. International Journal of Heat and Mass Transfer 42, 1501-1512.

Nicolaï, B., De Baerdemaeker, J., 1997. Finite element perturbation analysis of nonlinear heat conduction problems with random field parameters. International Journal of Numerical Methods for Heat \& Fluid Flow 7 (5), 525-544.

Nicolaï, B., Verboven, P., Scheerlinck, N., De Baerdemaeker, J., 1998. Numerical analysis of the propagation of random parameter fluctuations in time and space during thermal food processes. Journal of Food Engineering 38 (3), 259-278.

Nicolaï, B., Scheerlinck, N., Verboven, P., De Baerdemaeker, J., 2000. Stochastic perturbation analysis of thermal food processes with random field parameters. Transactions of the ASAE 43 (1), 131-138.

Pavliotis, G.A., Stuart, A.M., 2008. Multiscale methods,, .. Averaging and Homogenization, first ed. Springer Science+Business Media, LCC, New York. 
Perrot, N., Trelea, I.C., Baudrit, C., Trystram, G., Bourgine, P., 2011. Modeling and analysis of complex food systems: state of the art and new trends. Trends in Food Science \& Technology 22 (6), 304-314.

Porter, M.L., Schaap, M.G., Wildenschild, D., 2009. Lattice-Boltzmann simulations of the capillary pressure-saturation-interfacial area relationship for porous media. Advances in Water Resources 32 (11), 1632-1640.

Rakesh, V., Datta, A.K. (accepted for publication). Transport in deformable hygroscopic porous media during microwave puffing. AIChE Journal. (do 10.1002/aic.13793).

Rao, M.A., Rizvi, S.S.H., Datta, A.K., 2005. Engineering Properties of Foods, third ed. Taylor \& Francis, Boca Raton.

Russ, J.C., 2004. Image Analysis of Food Microstructure, first ed. CRC Press, Boca Raton, Florida.

Sablani, S., Datta, A.K., Rahman, M.S., Mujumdar, A.S., 2007. Handbook of Food and Bioprocess Modeling Techniques. CRC Press, Boca Raton, Florida.

Sahin, S., Sumnu, S.G., 2006. Physical Properties of Foods, first ed. SpringerTechnology \& Engineering, New York.

Scheerlinck, N., Verboven, P., Stigter, J., De Baerdemaeker, J., Van Impe, J., Nicolaï, B., 2000. Stochastic finite element analysis of coupled heat and mass transfer problems with random field parameters. Numerical Heat Transfer Part B Fundamentals 37 (3), 309-330.

Schrefler, B.A., 2004. Multiphase flow in deforming porous material. International Journal for Numerical Methods in Engineering 60 (1), 27-50.

Seo, Y., Datta, A.K., McCarthy, K.L., McCarthy, M.J., 2010. Heat transfer during microwave combination heating: computational modeling and MRI experiments. AIChE Journal 56, 2468-2478.

Sholokhova, Y., Kim, D., Lindquist, W.B., 2009. Network flow modeling via latticeBoltzmann based channel conductance. Advances in Water Resources 32 (2) 205-212.

Swift, M.R., Orlandini, E., Osborn, W.R., Yeomans, J.M., 1996. Lattice Boltzmann simulations of liquid-gas and binary fluid systems. Physical Review E 54 (5), 5041-5052.

Tanikawa, W., Shimamoto, T., 2009. Comparison of Klinkenberg-corrected gas permeability and water permeability in sedimentary rocks. International Journal of Rock Mechanics and Mining Sciences 46 (2), 229-238.

Tijskens, E., Ramon, H., De Baerdemaeker, J., 2003. Discrete element modeling for process simulation in agriculture. Journal of Sound and Vibration 266 (3), 493514.

Ubbink, J., Burbidge, A., Mezzenga, R., 2008. Food structure and functionality: a soft matter perspective. Soft matter 4 (8), 1569-1581.

Vafai, K., 2000. Handbook of Porous Media, first ed. Marcel Dekker Inc., New York

van der Graaf, S., Nisisako, T., Schroën, C.G.P.H., van der Sman, R.G.M., Boom, R.M. 2006. Lattice Boltzmann simulations of droplet formation in a T-shaped microchannel. Langmuir 22 (9), 4144-4152.

van der Sman, R.G.M., 1999. Solving the vent hole design problem for seed potato packagings, with the lattice Boltzmann scheme. International Journal of Computational Fluid Dynamics 11 (3-4), 237-248.

van der Sman, R.G.M., 2006. Galilean invariant lattice Boltzmann scheme for natural convection on square and rectangular lattices. Physical Review E 74 (2), art.nr 026705

van der Sman, R.G.M., 2007a. Soft condensed matter perspective on moisture transport in cooking meat. AIChE Journal 53 (11), 2986-2995.

van der Sman, R.G.M., 2007b. Lattice Boltzmann simulation of microstructures. Food Science and Technology 166, 15-40.

van der Sman, R.G.M., 2008. Prediction of enthalpy and thermal conductivity of frozen meat and fish products from composition data. Journal of Food Engineering 84 (3), 400-412.

van der Sman, R.G.M., 2009. Simulations of confined suspension flow at multiple length scales. Soft Matter 5 (22), 4376-4387.

van Der Sman, R.G.M., 2010. Drag force on spheres confined on the center line of rectangular microchannels. Journal of Colloid and Interface Science 351 (1), 43 49.

van der Sman, R.G.M., 2012. Effects of confinement on hydrodynamic interactions of suspensed spheres. Computers \& Fluids 58, 63-69.

van der Sman, R.G.M., Boer, E., 2005. Predicting the initial freezing point and water activity of meat products from composition data. Journal of Food Engineering $66,469-475$.

van der Sman, R.G.M., Broeze, J., 2011. Multiscale model of structure development in expanded starch snacks. In: Proceedings of the 11th International Congress on Engineering and Food (ICEF), pp. 2

van der Sman, R.G.M., Ernst, M., 2000. Convection-diffusion lattice Boltzmann scheme for irregular lattices. Journal of Computational Physics 160 (2), 766-782. van der Sman, R.G.M., Meinders, M., 2010. Prediction of the state diagram of starch water mixtures using the Flory-Huggins free volume theory. Soft Matter 7 (2), 429-442.

van der Sman, R.G.M., Van der Goot, A., 2008. The science of food structuring. Soft Matter 5 (3), 501-510.

van der Sman, R.G.M., van der Graaf, S., 2006. Diffuse interface model of surfactant adsorption onto flat and droplet interfaces. Rheologica Acta 46 (1), 3-11.

van der Sman, R.G.M., Vollebregt, H., Mepschen, A., Noordman, T.R., 2012. Review of hypotheses for fouling during beer clarification using membranes. Journal of Membrane Science 396, 22-31.

Van Liedekerke, P., Ghysels, P., Tijskens, E., Samaey, G., Roose, D., Ramon, H., 2011. Mechanisms of soft cellular tissue bruising. A particle base simulation approach. Soft Matter 7, 3580-3591.

Van Zeebroeck, M., Tijskens, E., Dintwa, E., Kafashan, J., Loodts, J., De Baerdemaeker, J., Ramon, H., 2006a. The discrete element method (DEM) to simulate fruit impact damage during transport and handling: model building and validation of DEM to predict bruise damage of apples. Postharvest Biology and Tchnology 41 (1), 85-91.

Van Zeebroeck, M., Tijskens, E., Dintwa, E., Kafashan, J., Loodts, J., De Baerdemaeker, J., Ramon, H., 2006b. The discrete element method (DEM) to simulate fruit impact damage during transport and handling: case study of vibration damage during apple bulk transport. Postharvest Biology and Technology 41 (1), 92100.

Veraverbeke, E., Verboven, P., Van Oostveldt, P., Nicolaï, B., 2003a. Prediction of moisture loss across the cuticle of apple (Malus sylvestris subsp mitis (Wallr.)) during storage Part 1 . Model development and determination of diffusion coefficients. Postharvest Biology and Technology 30 (1), 75-88.

Veraverbeke, E., Verboven, P., Van Oostveldt, P., Nicolaï, B., 2003b. Prediction of moisture loss across the cuticle of apple (Malus sylvestris subsp mitis (Wallr.)) during storage: part 2. Model simulations and practical applications. Postharvest Biology and Technology 30 (1), 89-97.

Verboven, P., Kerckhofs, G., Mebatsion, H., Ho, Q., Temst, K., Wevers, M., Cloetens, P., Nicolaï, B., 2008. Three-dimensional gas exchange pathways in pome fruit characterized by synchrotron X-ray computed tomography. Plant Physiology 147 (2) , 518-527.

Verstreken, E., Van Hecke, P., Scheerlinck, N., De Baerdemaeker, J., Nicolaï, B., 1998. Parameter estimation for moisture transport in apples with the aid of NMR imaging. Magnetic Resonance in Chemistry 36 (3), 196-204.

Vollebregt, H., van der Sman, R.G.M., Boom, R.M., 2010. Suspension flow modelling in particle migration and microfiltration. Soft Matter 6 (24), 6052-6064.

von der Schulenburg, D., Pintelon, T., Picioreanu, C., Van Loosdrechtet, M.C.M., Johns, M.L., 2009. Three-dimensional simulations of biofilm growth in porous media. AIChE Journal 55 (2), 494-504.

Wallach, R., Troygot, O., Saguy, I.S., 2011. Modeling rehydration of porous food materials: II. The dual porosity approach. Journal of Food Engineering 105, 416421.

Wang, Y., Brasseur, J.G., Banco, G.G., Webb, A.G., Ailiani, A.C., Neuberger, T., 2010. A multiscale lattice Boltzmann model of macro-to micro-scale transport, with applications to gut function. Philosophical Transactions of the Royal Society A: Mathematical, Physical and Engineering Sciences 368 (1921), 2863-2880.

Weerts, A.H., Lian, G., Martin, D.R., 2003. Modeling the hydration of foodstuffs: temperature effects. AIChE Journal 49 (5), 1334-1339.

Whitaker, S., 1977. Simultaneous heat, mass, and momentum transfer in porous media: a theory of drying. Advances in Heat Transfer 13, 119-203.

Whittle, M., Dickinson, E., 2001. On simulating colloids by dissipative particle dynamics: issues and complications. Journal of Colloid and Interface Science 242 (1), 106-109.

Yamsaengsung, R., Moreira, R.G., 2002. Modeling the transport phenomena and structural changes during deep fat frying - Part 1: model development. Journal of Food Engineering 53 (1), 1-10.

Yue, X., E, W., 2007. The local microscale problem in the multiscale modeling of strongly heterogeneous media: effects of boundary conditions and cell size. Journal of Computational Physics 222 (2), 556-572.

Zhang, D.X., Zhang, R.Y., Chen, S.Y., Soll, V.E., 2000. Pore scale study of flow in porous media: scale dependency, REV, and statistical REV. Geophysical Research Letters 27, 1195-1198.

Zhang, J., Datta, A.K., Mukherjee, S., 2005. Transport processes and large deformation during baking of bread. AIChE Journal 51 (9), 2569-2580.

Zienkiewicz, O.C., Taylor, R.L., 2005. The Finite Element Method. sixth ed. Butterworth-Heinemann, Oxford.

Zygalakis, K.C., Kirk, G.J.D., Jones, D.L., Wissuwa, M., Roose, T., 2011. A dual porosity model of nutrient uptake by root hairs. New Phytologist 192, 676-688. 